Les sources de l'Histoire des Mines : Nouveaux outils, Nouvelles approches

\title{
Inaki Reguera, Ramon OJEDA, Eduardo Alonso, Victor Arroyo, José Maria Beascoecha, Arsenio Dacosta, Rialia Museo de la industria
}

Portugalete, Casa del Mar, Pays basque espagnol, 2006, 150 pages., ill. en noir et blanc et en couleurs.

\section{Véronica Tello}

\section{OpenEdition}

Édition électronique

URL : http://journals.openedition.org/dht/736

DOI : $10.4000 /$ dht.736

ISSN : 1775-4194

Éditeur :

Centre d'histoire des techniques et de l'environnement du Cnam (CDHTE-Cnam), Société des élèves du CDHTE-Cnam

\section{Édition imprimée}

Date de publication : 1 décembre 2008

Pagination : 229-231

ISBN : 978-2-95-30779-2-6

ISSN : 0417-8726

Référence électronique

Véronica Tello, «Inaki Reguera, Ramon OJeDA, Eduardo Alonso, Victor Arroyo, José Maria Beascoecha, Arsenio Dacosta, Rialia Museo de la industria ", Documents pour I'histoire des techniques [En ligne], 16। $2^{\mathrm{e}}$ semestre 2008, mis en ligne le 05 octobre 2010, consulté le 24 septembre 2020. URL : http:// journals.openedition.org/dht/736 ; DOI : https://doi.org/10.4000/dht.736

Ce document a été généré automatiquement le 24 septembre 2020.

(c) Tous droits réservés 


\section{Inaki Reguera, Ramon OJEDA,} Eduardo Alonso, Victor Arroyo, José Maria Beascoecha, Arsenio Dacosta, Rialia Museo de la industria

Portugalete, Casa del Mar, Pays basque espagnol, 2006, 150 pages., ill. en noir et blanc et en couleurs.

\section{Véronica Tello}

\section{RÉFÉRENCE}

Inaki Reguera, Ramon OJEDA, Eduardo Alonso, Victor Arroyo, José Maria Beascoecha, Arsenio Dacosta, Rialia Museo de la industria, Portugalete, Portugalete, Casa del Mar, Pays basque espagnol, 2006, 150 pages., ill. en noir et blanc et en couleurs.

1 À l'occasion de l'ouverture du Musée Rialia ${ }^{1}$, et suite à l'inscription sur la Liste du patrimoine mondial de l'UNESCO du pont transbordeur de Vizcaya (pont transbordeur monumental reliant la ville de Portugalete à la ville de Gotxa), la Mairie de Portugalete associée au Musée, au Ministère de la culture espagnol, à la SEPI (Société de l'État de participation industrielle) et aux SURPOSA (Servicios de urbanismo y rehabilitacion Portugalete sociedad anonima) a édité cet ouvrage dont la vocation est de rendre compte, par un travail de muséographie et d'animation, de la mémoire du premier centre sidérurgique d'Espagne à fin du XIX ${ }^{\mathrm{e}}$ siècle.

Trois acteurs forment la source d'inspiration principale de ce projet muséographique : l'homme, l'industrie et la ria (le bras du fleuve), tous étroitement liés. Si une place particulière est accordée aux conditions géographiques, ce catalogue d'exposition au riche support historiographique est surtout conçu comme un hommage aux hommes et femmes à l'origine de cette épopée industrielle. Il s'organise autour de six sujets, qui sont en principe le reflet du contenu thématique des salles d'exposision. 
3 Ainsi la premiere partie du catalogue assurée par Inaki Reguera donne les clés de l'origine de la ville de Portugalete fondée en 1322, date à laquelle la Dame de Bizcaye, “ dona Maria Diaz de Haro", signe "una carta-puebla fundacional" accordant aux habitants du hameau la qualité de résidents d'une "villa" (village au statut noble, par rapport à un hameau), avec privilèges politiques et économiques. La lecture de ce chapitre est fluide, donnant un aperçu du contexte local et national jusqu'à l'époque contemporaine. Bien qu'abondamment illustré en photographies noir et blanc, provenant des différentes archives, et réalisées pour l'occasion, le manque de document cartographique et ou graphique antérieur au XIXe siècle est néanmoins à déplorer.

4 Ramon Ojeda San Miguel, dans le chapitre dédié au "Temps de l'industrialisation" précise que le réel décollage industriel et économique de la sidérurgie de Bizcaye intervient après la fin de la Guerre carliste (1841) avec d'une part, sur ordonnance du pouvoir central, le déplacement du système douanier, anciennement installé à l'intérieur du pays et désormais placé sur le littoral basque, notamment sur la ria du Nervion, et d'autre part, l'adoption, dans les années 1870 et 1880 , des évolutions techniques du procédé Bessemer (patenté en 1856) et un peu plus tard de celui de Martin-Siemens : deux procédés qui permettent une plus grande (et moins coûteuse) production de l'acier et réduisent la dépendance envers le charbon provenant de l'Asturie.

5 Ainsi dans les années 1880, la production des lingots d'acier Bessemer, soutenue et protégée par l'État, donne à la Bizcaye une hégémonie nationale sans conteste. La capacité de production de la région dépasse la consommation du pays qui, à cette époque, importe une bonne partie de ses besoins (50\% environ). L'État espagnol impose alors une politique de "substitution des importations", consolidant de cette façon le pouvoir industriel et financier du nord de la péninsule ibérique. Tous ces processus favorisent l'apparition, à l'image de certains modèles bancaires dèjà confirmés dans les pays industrialisés européens, d'un nouveau type de capital: monopolistique, expansioniste et protégé par l' État. L'ensemble du texte est illustré de façon cohérente de photographies d'archives en noir et blanc ainsi que de quelques dessins panoramiques de 1943, magnifiques de précision. La page de garde de chaque chapitre est pourvue d'une photographie du paysage industriel et urbain de haute qualité esthétique, en grand format et en couleurs.

Ce chapitre aurait pu introduire celui consacré au rôle joué par la Banca en Vizcaya dans le processus d'industrialisation durant la période de 1857 à 1903. Mais les auteurs du catalogue nous présentent en lieu et place un volet consacré aux entrepreneurs de Biscaye entre 1840 et 1940 par Eduardo J.Alonso Olea, ce qui rend la lecture moins aisée qu'on pourrait l'envisager. L'auteur nous propose une série de biographies très bien documentées de personnages impliqués dans cette aventure. Il met en évidence les caractéristiques communes de ces personnages et familles que Miguel de Unamuno avait défini comme les "comtes de la sidérurgie". En réalité, il s'agit, à l'exception de quelques ingénieurs, de commerçants déjà familiarisés avec le commerce du fer, de la laine et des étoffes, nés entre la fin du XVIII et XIXe siècle, possédant un certain capital et cherchant à amplifier leur activités vers des nouveaux horizons. Ces biographies sont illustrées par des photographies d'archives des sites mais il est étonnant de ne voir restitué aucun portrait de ces protagonistes du développement du XIX ${ }^{e}$ siècle au Pays basque espagnol, alors que les salles du Musée donnent un aperçu assez éloquent de cet 
aspect de l'histoire de la sidérurgie en Bizcaye, à travers des portraits peints et des archives photographiques.

7 Le catalogue propose ensuite un retour sur le capitalisme industriel et bancaire avec la naissance des institutions financières dans le nord de l'Espagne. J. Victor Arroyo Martin décrit méthodiquement cette "nouvelle modernité" industrielle, sociale et économique. La création de la banque étroitement associée à la métallurgie et aux secteurs d'activité dérivés constitue la source principale de cette modernité : elle émet les premiers billets de banque, action révolutionnaire, en remplacement de la monnaie traditionnelle et, surtout, à l'initiative d'un phénomène sans précédent d'épargne populaire, lié à l'apparition des nouvelles couches sociales industrieuses et industrielles. Capter de façon régulière le passif monétaire de nouveaux clients donne à la Banque de Bilbao un pouvoir supplémentaire : celui de "réactiver" cet argent, notamment dans le secteur de l'industrie régionale. La suite du sujet traite du parcours, émaillé de hauts et de bas, des institutions financières et industrielles du Pays Basque espagnol.

8 L'avant dernier chapitre du catalogue traite de la formation d'un axe urbain autour de la ria aux $\mathrm{XIX}^{\mathrm{e}}$ et $\mathrm{XX}^{\mathrm{e}}$ siècles. José Maria Beascoechea Gangoiti met en évidence l'influence du fleuve sur la configuration urbaine et industrielle depuis la ville de Bilbao jusqu'à Portugalete, à l'embouchure. Explosion démographique, activités minières, activités portuaires, industrie métallurgique et commerce sont décrits de façon minutieuse de même que les caractéristiques naturelles des rives: marais, zones inondables et plages qui peu à peu sont envahis par l'industrie, le «ferrocarril » (chemin de fer) et les ports, sans réelle planification. Beaucoup d'images en noir et blanc illustrent ce récit, sans que l'on ait pour autant un aperçu territorial de cette évolution par la cartographie, des plans, des vues des territoires, etc.

9 En conclusion du catalogue d'exposition Rialia Museo de la industria, Portugalete, Arsenio Dacosta divulgue la "Coleccion Altos Hornos de Vizcaya », importante collection de toiles représentant des paysages industriels et des scènes de travail à l'intérieur des usines, d'une facture picturale remarquable, réunie par le Musée de l'industrie Rialia, et support de la muséologie. L'auteur, dans cet article intitulé « Notes à propos de l'histoire d'une collection d'art de type corporatiste en Biscaye ", questionne le lecteur aussi bien que le visiteur du Musée et de la ville de Portugalete sur les motivations et le bien fondé d'une telle collection. Loin d'apporter une réponse, Arsenio Dacosta nous éclaire sur d'autres aspects non moins intéressants, comme l'unité thématique choisie par les commanditaires, la cohérence des formats, l'importance des signatures (artistes) et la durée dans le temps de la constitution de cette collection. En effet, cette initiative de la fin du XIX ${ }^{\mathrm{e}}$ siècle, attitude profondément avant-gardiste pour l'époque, prends une consistance particulière et devient une constante au moment de la fusion qui donne lieu, en 1902, à « la grande corporation industrielle» du Pays basque. Dans un premier temps, ces acquisitions picturales servent au décor du siège de AHV (Altos Hornos de Vizcaye) situé à Bilbao, près du Musée des Beaux arts et du Grand théâtre, en étroite relation avec le lieu selon Arsenio Dacosta. Pour l'auteur, cette collection dès l'origine, répond à une préoccupation autre que la richesse engendrée par l'industrie métallurgique, sans que la réelle vocation de cette démarche ait été clairement formulée : s'agissait-il de rechercher de nouvelles valeurs ou de revendiquer d'autres identités?

C'est dans cette partie du catalogue, parallèlement à la question de l'identité, que la question du patrimoine est évoquée comme une autre forme de collection, notamment 
par la présentation du bâtiment qui abrite le Musée et le fameux Pont Transbordeur : « entrée monumentale de la ria, il est porteur d'une fierté basque importante en tant que première réalisation de ce type d'ouvrage et par la personnalité du concepteur du pont transbordeur, Alberto de Palacio » selon Michel Cotte ${ }^{2}$. Le pont transbordeur de Vizcaya a été inscrit sur la Liste du patrimoine mondial en 2006. L'ensemble des peintures, représentées en couleurs dans le catalogue, accompagne à merveille ces derniers propos. Cette collection signée d'artistes espagnols, allemands et français dont certaines toiles, notamment celles qui représentent les activités humaines dans l'industrie de l'acier, retiennent longuement l'attention. C'est le cas de Convertidores de acero Bessemer, signé R. Gurrea, et des grands formats de Luna reproduisant les hauts fourneaux, les scènes de travail et la coulée à l'intérieur des ateliers d'acier dits Robert.

$11 \mathrm{Au}$ final, le contenu de ce catalogue, à l'exception du dernier chapitre, est une mine de renseignements historiques sur le sujet et bien que d'une lecture aisée, semble plutôt adapté aux chercheurs qu'au grand public. Il est un peu difficile de savoir, à distance, s'il est conçu comme une suite au parti-pris muséographique ou bien l'inverse. Néanmoins, la dernière partie du catalogue d'exposition Rialia Museo de la industria, Portugalete, ouvrage très documenté, nous réconcilie avec sa vocation première: les hommes et les femmes, acteurs de ce pan de l'histoire industrielle du Pays basque. La ria, la ville, les œuvres d'art et le pont transbordeur: voilà une invitation qui ne manque pas d'intérêt.

\section{NOTES}

1. Site internet du Musée : www.rialia.net

2. Michel Cotte, «Le pont transbordeur de Vizcaya à Bilbao. Du projet novateur d'hier au patrimoine d'aujourd'hui », communication au Premier Congrès francophone d'histoire de la construction, Paris, CDHTE-Cnam, 19-21 juin 2008. Nous remercions vivement M. Michel Cotte de nous avoir autorisée à présenter ses recherches.

\section{AUTEURS}

\section{VÉRONICA TELLO}

CDHTE-Cnam 